\title{
On Discontinuity and Tail Behaviours of the Integrated Density of States for Nested Pre-fractals
}

\author{
Masatoshi Fukushima ${ }^{\star}$, Tadashi Shima ${ }^{\star}$ \\ Department of Mathematical Science, Faculty of Engineering Science, Osaka University, \\ Toyonaka, Osaka 560, Japan. Fax: 06(857)-0253. E-mail`: fuku@sigmath.osaka-u.ac.jp \\ $\star \star$ : tadashi@sigmath.osaka-u.ac.jp
}

Received: 30 April 1993

\begin{abstract}
We consider a general finitely ramified fractal set called a nested fractal which is determined by $N$ number of similitudes. Basic properties of the integrated density of states $\mathscr{N}(x)$ for the discrete Laplacian on the associated nested prefractal are investigated. In particular $d \mathscr{N}$ is shown to be purely discontinuous if $M<N$, where $M$ is the number of branches of the inverse of the rational function involved in the spectral decimation method due to Rammal-Toulouse. Sierpinski gaskets and the modified Koch curve are special examples.
\end{abstract}

\section{Introduction}

The integrated density of states (IDS in abbreviation) is defined as the limit of the normalized distribution function of the eigenvalues of $-\Delta$ when the size of the underlying space is made to expand to infinity. If the underlying space is a domain of the Euclidean space $R^{d}$ or a finite subset of the lattice $Z^{d}$, then the IDS is known to be absolutely continuous and behave like $C x^{d / 2}$ as $x \downarrow 0$. The present paper will concern the cases where the underlying spaces are in a general class of finitely ramified fractal sets called nested fractals by Lindstrøm [7]. The discrete Laplacian (a certain difference operator) on the nested pre-fractal and the Laplacian $\Delta$ on the nested fractal are now well defined objects $[2,6,7]$.

The Sierpinski gasket is a typical example of the nested fractal. Rammal [10] considered the discrete Laplacian on the Sierpinski pre-gasket located in $R^{d}, d \geqq 2$, and discovered that its IDS is purely discontinuous. Fukshima-Shima [4] proved the same property of IDS of the Laplacian on the Sierpinski gasket in $R^{d}, d \geqq 2$. In both cases, the IDS can be described explicitly owing to the spectral decimation method due to Rammal-Toulouse [11], which relates eigenvalues of the successive pre-gaskets by the inverse function of a certain quadratic function. Recently Malozemov $[8,9]$ found that the modified Koch curve also admits the spectral decimation with respect to a certain rational function and that the IDS for the corresponding discrete Laplacian is purely discontinuous.

In this paper we consider a general nested fractal and study the IDS $\mathscr{N}(x)$ of the discrete Laplacian on the corresponding nested pre-fractal (rather than the 
Laplacian on the nested fractal itself). Let the nested fractal be decided by $N$ number of $\alpha$-similitudes $(\alpha>1)$. We first clarify under what circumstances $d \mathscr{N}$ becomes discontinuous. To this end, we assume that the nested fractal admits the spectral decimation with respect to a rational function $\Phi(x)$. Let $M$ be the number of branches of the inverse function of $\Phi$. In the next section, we show that $M \leqq N$ in general. We further prove the following:

1. If $M<N$, then $d \mathscr{N}$ is purely discontinuous.

2. If $M=N$ and $\Phi$ is a polynomial, then $d \mathscr{N}$ is the Brolin measure [1], namely, the logarithmic equilibrium measure of the Julia set of the transformation $\Phi$ on the complex plane.

The Sierpinski gasket on $R^{d}$ with $d \geqq 2$ and the modified Koch curve studied by Malozemov are certainly examples of case 1 . The one-dimensional interval can be viewed as a Sierpinski gasket and the corresponding (infinite) pre-gasket is identified with $Z^{1}$. This turns out to be an example of case 2 . The corresponding Julia set is a real interval on the complex plane and we recover the well known absolutely continuous IDS of the discrete Laplacian on $Z^{1}$ as the logarithmic equilibrium measure for this Julia set.

In Sect. 3, we shall prove that the IDS $\mathscr{N}(x)$ behaves like $C x^{d_{s} / 2}$ as $x \downarrow 0$, where $d_{s}$ is the spectral dimension. This has been shown in Fukushima [2] for the IDS for the nested fractal, which was easier to handle than the present pre-fractal case because a simpler scaling property of the Dirichlet norm was available. See Malozemov [9] for a finer tail behaviour of IDS for the discrete Laplacian in case of the modified Koch graph.

\section{Discontinuity of IDS for the Nested Pre-fractal}

Let $\Phi$ be a rational function with real coefficients and $M$ be the number of branches of the inverse $\Phi^{-1}$. For $x, y \in R, x$ is called an $n^{\text {th }}$ predecessor of $y$ if $y=\Phi^{n}(x)$. We denote by $P_{y}^{(n)}$ the set of all $n^{\text {th }}$ predecessors of $y$. We let $P_{y}^{(0)}=\{y\}$. We say that $y \in R$ is proper for $\Phi$ if $\# P_{y}^{(n)}=M^{n}, n=1,2, \ldots$.

For $\alpha>1$, a mapping $\Psi$ from $R^{d}$ to $R^{d}$ is said to be an $\alpha$-similitude if $\Psi x=\alpha^{-1} U x+\beta, x \in R^{d}$, for some unitary map $U$ and $\beta \in R^{d}$. Given a collection $\Psi=\left\{\Psi_{1}, \Psi_{2}, \ldots, \Psi_{N}\right\}$ of $\alpha$-similitudes, we let

$$
\Psi(A)=\bigcup_{i=1}^{N} \Psi_{i}(A), \quad A \subset R^{d} .
$$

There exists then a unique compact set $E \subset R^{d}$ such that $\Psi(E)=E$. The pair $(\Psi, E)$ is called a self similar fractal.

For $A \subset R^{d}$ and integer $n \geqq 1$, we let

$$
\begin{aligned}
& A_{i_{1}} \cdots i_{n}=\Psi_{i_{1}} \cdots \Psi_{i_{n}}(A), \quad 1 \leqq i_{1}, \ldots, i_{n} \leqq N, \\
& A^{(n)}=\Psi^{(n)}(A)=\underset{1 \leqq i_{1}, \ldots, i_{n} \leqq N}{\bigcup} A_{i_{1}} \cdots i_{n}, A^{(0)}=A .
\end{aligned}
$$

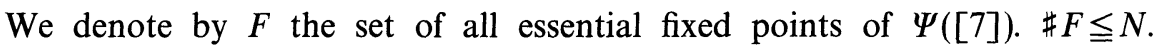
Lindstrøm [7] calls a self similar fractal $(\Psi, E)$ a nested fractal if three axioms (axioms of connectivity, symmetry and nesting) and the open set condition are 
fulfilled and $\# F \geqq 2$. We refer the readers to [7] for details but we note that the nesting axiom requires

$$
E_{i_{1}} \ldots i_{n} \cap E_{j_{1}} \ldots j_{n}=F_{i_{1}} \ldots i_{n} \cap F_{j_{1}} \ldots j_{n}\left(i_{1}, \ldots, i_{n}\right) \neq\left(j_{1}, \ldots, j_{n}\right) .
$$

which expresses the finite ramifiedness.

For a nested fractal $(\Psi, E)$ as above, we assume without loss of generality that $0 \in F$ and $\Psi_{1}=\alpha^{-1} x, x \in R^{d}$. $E$ equals the closure in $R^{d}$ of the countable set $F^{(\infty)}=\bigcup_{k=0}^{\infty} F^{(k)}$. In this paper however, we identify the finite set $F^{(k)}$ with its similar one $\tilde{F}^{(k)}=\alpha^{k} F^{(k)}$. Thus, rather than the bounded set $F^{(\infty)}$, we are thinking of the unbounded set

$$
\tilde{F}^{(\infty)}=\bigcup_{k=0}^{\infty} \tilde{F}^{(k)}
$$

which will be called the nested pre-fractal associated with the nested fractal $E$. Denote by $N_{k}$ the cardinality of the set $F^{(k)} \backslash F$. It is known that

$$
0<c_{1} \leqq \frac{N_{k}}{N^{k}} \leqq c_{2}<\infty
$$

for some constants $c_{1}, c_{2}$.

Let $P_{\eta \xi}^{(k)}$ be the transition probability of Lindstrøm random walk on $F^{(k)}\left(\sim \tilde{F}^{(k)}\right)$ absorbed at the boundary $F\left(\sim \alpha^{k} F\right)$, cf. [7]. Its generator $H_{k}$ (which we may call the discrete Laplacian) is defined by

$$
H_{k} u(\xi)=\sum_{\eta \in F^{(k)}} p_{\xi \eta}^{(k)}(u(\eta)-u(\xi)), \quad \xi \in F^{(k)} \backslash F
$$

with the boundary condition

$$
u=0 \text { on } F
$$

Denote by

$$
\mathscr{A}_{k}=\left\{\gamma_{1}, \gamma_{2}, \ldots, \gamma_{N_{k}}\right\}
$$

the collection of eigenvalues of $-H_{k}$ with the same value being repeated according to its multiplicity and let

$$
v_{k}=\frac{1}{N_{k}} \sum_{p=1}^{N_{k}} \delta_{\gamma_{p}}, \quad v=\lim _{k \rightarrow \infty} v_{k} .
$$

The limiting probability measure $v$ exists as we shall see in the next section. The non-decreasing function $\mathcal{N}(x)=v([0, x]), x \in R$ is called the integrated density of states (IDS) for the nested pre-practical $\widetilde{F}^{(\infty)}$.

Let $(\Psi, E)$ and $\Phi$ be a nested fractal and a rational function as above. We say that $(\Psi, E)$ admits a spectral decimation with respect to $\Phi$ if the counting measures $v_{k}$ of $\mathscr{A}_{k}, k=1,2, \ldots$, can be described as

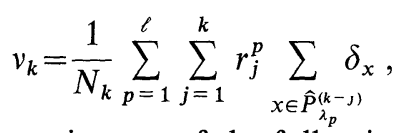

under the assumption of the existence of the following three objects:

$$
\text { non-negative numbers } \lambda_{1}, \lambda_{2}, \ldots, \lambda_{\ell} \text {, }
$$


which are distinct and proper for $\Phi$,

$$
\text { non-negative integers } r_{j}^{1}, r_{j}^{2}, \ldots, r_{j}^{\ell}, j=1,2, \ldots \text {, }
$$

and

$$
\text { a subset } \hat{P}_{\lambda_{p}}^{(j)} \text { of } P_{\lambda_{p}}^{(j)}, 1 \leqq p \leqq \ell,
$$

such that the cardinality $M_{p, j}$ of $\hat{P}_{\lambda_{p}}^{(j)}$ satisfies

$$
c_{3} M^{j} \leqq M_{p, j} \leqq M^{j}, \quad 1 \leqq p \leqq \ell, \quad j=1,2, \ldots,
$$

for some constant $c_{3}>0$.

We call the members of the set $\hat{P}_{\lambda_{p}}^{(j)}$ in the above the allowed $J^{\text {th }}$ predecessors of $\lambda_{p}$. The expression (4) means that, for each $p, 1 \leqq p<\ell, \lambda_{p}$ is a distinguished value appearing in the set $\mathscr{A}_{j}$ in each step $j$ but with multiplicity $r_{j}^{p}$, whose allowed $j^{\prime \text { th }}$ predecessors keep appearing in the successive sets $\mathscr{A}_{j+j^{\prime}}, j^{\prime}=1,2, \ldots$, with the same multiplicity $r_{j}^{p}$, and that the eigenvalues are exhausted in this way. At the end of this section, we shall give three examples in which this spectral decimation works.

It is convenient to rewrite the measure $v_{k}$ as follows:

$$
v_{k}=\frac{1}{N_{k}} \sum_{p=1}^{\ell} \sum_{j=1}^{k} r_{j}^{p} M_{p, k-j} \hat{v}_{\lambda_{p}}^{(k-j)},
$$

where

$$
\hat{v}_{\lambda_{p}}^{(j)}=\frac{1}{M_{p, j}} \sum_{\substack{x \in \hat{P}_{\lambda p}^{(j)} \\ \lambda_{p}}} \delta_{x} .
$$

the normalized counting measure of the set $\hat{P}_{\lambda_{p}}^{(j)}$ of the allowed $j^{\text {th }}$ predecessors of $\lambda_{p}$.

Theorem 1. Assume that $(\Psi, E)$ admits a spectral decimation with respect to $\Phi$.

(i) $M \leqq N$.

(ii) Suppose $M<N$, then for any non-negative Borel function $f$ on $R$,

$$
\langle v, f\rangle \leqq \frac{c_{2}}{c_{1}} \sum_{p=1}^{\ell} \bar{\alpha}_{p} \sum_{j=0}^{\infty}\left(\frac{M}{N}\right)^{j}\left\langle\hat{v}_{\lambda_{p}}^{(j)}, f\right\rangle,
$$

where

$$
\bar{\alpha}_{p}=\varlimsup_{k \rightarrow \infty} \frac{r_{k}^{p}}{N_{k}} \quad(\leqq 1), 1 \leqq p \leqq \ell
$$

In particular, $\bar{\alpha}_{p}>0$ for some $p, 1 \leqq p \leqq \ell$, and the measure $v$ is concentrated on the countable set $\bigcup_{p=1}^{\ell} \bigcup_{j=1}^{\infty} \hat{P}_{\lambda_{p}}^{(j)}$. Furthermore, if the limits

$$
\lim _{k \rightarrow \infty} \frac{N_{k}}{N^{k}}=c_{0}, \quad \lim _{k \rightarrow \infty} \frac{r_{k}^{p}}{N_{k}}=\alpha_{p}, 1 \leqq p \leqq \ell
$$

exist, then

$$
v=\sum_{p=1}^{\ell} \alpha_{p} \sum_{f=0}^{\infty} \frac{M_{p, j}}{N^{j}} \hat{v}_{\lambda_{p}}^{(j)} .
$$


(iii) Suppose $M=N, \Phi$ is a polynomial and $\hat{P}_{\lambda_{p}}^{(j)}=P_{\lambda_{p}}^{(j)}, \forall p, \forall j$, then $v$ equals the Brolin measure for $\Phi$.

Proof. First note that (5) implies the identity

$$
N_{k}=\sum_{p=1}^{\ell} \sum_{j=0}^{k} r_{k-j}^{p} M_{p, j}=\sum_{p=1}^{\ell} \sum_{j=1}^{k} r_{j}^{p} M_{p, k-j}
$$

holding for all $k$.

(i) Suppose $M>N$. We see from $N_{1}=\sum_{p=1}^{\ell} r_{1}^{p} M_{p, 1}$ that at least one of $r_{1}^{p}$ 's, say $r_{1}^{1}$ is positive and

$$
\frac{N_{k}}{N^{k}} \geqq r_{1}^{1} \frac{M_{1, k-1}}{N^{k}} \geqq r_{1}^{1} \frac{c_{3} M^{k-1}}{N^{k}},
$$

which tends to infinity as $k \rightarrow \infty$, a contradiction.

(ii) Suppose $M<N$. For any $\varepsilon>0$, there then exists $L_{0}$ such that for any $k$ and $L$ with $k>L>L_{0}$,

$$
\frac{1}{N_{k}} \sum_{p=1}^{\ell} \sum_{j=L}^{k-1} r_{k-j}^{p} M_{p, j}<\varepsilon
$$

In fact, the left side of (9) equals

$$
\begin{aligned}
\frac{1}{N_{k}} \sum_{p=1}^{\ell} \sum_{j=1}^{k-L} r_{j}^{p} M_{p, k-j} & =\frac{N_{k-L}}{N_{k}} \cdot \frac{1}{N_{k-L}} \sum_{p=1}^{\ell} \sum_{j=1}^{k-L} r_{j}^{p} M_{p, k-j-L} \cdot \frac{M_{p, k-j}}{M_{p, k-j-L}} \\
& \leqq \frac{c_{2}}{c_{1}} \frac{1}{N^{L}} \cdot \frac{1}{c_{3}} M^{L}=\frac{c_{2}}{c_{1} c_{3}}\left(\frac{M}{N}\right)^{L},
\end{aligned}
$$

which can be made arbitrarily small for large $L$.

For any continuous function $f$ on $R$ such that $0 \leqq f \leqq 1$, we get from (5) and (9),

$$
\begin{aligned}
\left\langle v_{k}, f\right\rangle & \leqq \frac{1}{N_{k}} \sum_{p=1}^{\ell} \sum_{j=0}^{L-1} r_{k-j}^{p} M_{p, j}\left\langle\hat{v}_{\lambda_{p}}^{j}, f\right\rangle+\varepsilon \\
& \leqq \frac{c_{2}}{c_{1}} \sum_{p=1}^{\ell} \sum_{j=0}^{L-1} \frac{r_{k-j}^{p}}{N_{k-j}}\left(\frac{M}{N}\right)^{j}\left\langle\hat{v}_{\lambda_{p}}^{j}, f\right\rangle+\varepsilon .
\end{aligned}
$$

It is enough to let $k \rightarrow \infty, L \rightarrow \infty, \varepsilon \downarrow 0$ in this order to get the inequality (6). The latter assertion of (ii) can be proved similarly.

(iii) Under the stated assumption, $M_{p, j}=N^{j}$ and $\hat{v}_{\lambda_{p}}^{(j)}=v_{\lambda_{p}}^{(j)}$ the normalized counting measure of the $j^{\text {th }}$ predecessors of $\lambda_{p}$. Note that the set $P_{\lambda_{p}}^{(j)}$ exhausts all $j^{\text {th }}$ (complex) predecessors of $\lambda_{p}$ with respect to the $N^{\text {th }}$ order polynomial $\Phi$ considered as a transformation on the extended complex plane. Hence the Julia set $J(\subset C)$ of $\Phi$ in the sense of Brolin coincides with the set of all accumulation points of $\bigcup_{j=1}^{\infty} P_{\lambda_{p}}^{(j)}$ for each $p$ (see Theorem 2.5 of $[1] ; \lambda_{p}$ 's are different from the exceptional values in the statement of Theorem 2.5 of [1] because, otherwise, $\# P_{\lambda_{p}}^{(j)}=1$ in view of the proof of Lemma 2.2 of [1]). In particular, $J$ must be a subset of the real line.

Denote by $v^{*}$ the logarithmic equilibrium measure for the Julia set $J$. We call $v^{*}$ the Brolin measure for $\Phi$. On account of Theorem 16.1 of [1], the measure $v_{\lambda_{p}}^{j}$ converges as $j \rightarrow \infty$ to $v^{*}$ for each $p$. 
Let us put $r_{j}=\sum_{p=1}^{\ell} r_{j}^{p}$. We have from (8) the bound

$$
\frac{c_{2}}{c_{1}} \geqq \frac{N_{k}}{N^{k}}=\sum_{j=1}^{k} \frac{r_{j}}{N^{j}}
$$

and the infinite sum of $\frac{r_{j}}{N^{j}}$ is covergent. In particular

$$
\lim _{k \rightarrow \infty} \frac{r_{k}}{N^{k}}=0
$$

For any continuous function on $R$ with $\|f\|_{\infty} \leqq 1$, we let $a_{p}^{j}=\left\langle v_{\lambda_{p}}^{(j)}, f\right\rangle$ and $a^{*}=\left\langle v^{*}, f\right\rangle$. For any $\dot{\varepsilon}>0$, there exists $L$ such that, for any $j \geqq L,\left|a_{p}^{j}-a^{*}\right|<\varepsilon$. We then have by (5),

$$
\begin{aligned}
\left|\left\langle v_{k}, f\right\rangle-a^{*}\right| & \leqq \frac{1}{N_{k}} \sum_{p=1}^{\ell} \sum_{j=0}^{k-1} r_{k-j}^{p} M_{p, j}\left|a_{p}^{j}-a^{*}\right| \\
& \leqq 2 \frac{1}{N_{k}} \sum_{p=1}^{\ell} \sum_{j=0}^{L-1} r_{k-j}^{p} M_{p, j}+\varepsilon \\
& \leqq \frac{2}{c_{1}} \sum_{j=0}^{L-1} \frac{r_{k-j}}{N^{k-j}}+\varepsilon .
\end{aligned}
$$

(10) then implies $\left|\langle v, f\rangle-a^{*}\right|<\varepsilon$.

Example 1 (Sierpinski gasket). Let $(\Psi, E)$ be the Sierpinski gasket in $R^{N-1}(N \geqq 3)$. $\Psi$ consists of $N$-number of 2 -similitudes. $N_{k}=\frac{N}{2}\left(N^{k}-1\right) . H_{k}$ is given by

$$
\left.H_{k} u(\xi)=\frac{1}{2(N-1)} \sum_{\eta \in F_{k, \zeta}}(u(\eta))-u(\xi)\right), \xi \in F_{k} \backslash F,
$$

where $F_{k, \xi}$ denotes the 2(N-1) neighbours of $\xi$. As was proven by Shima [12], the spectral decimation works with the quadratic function

$$
\Phi(x)=x(N+2-2(N-1) x) .
$$

Hence $M=2(<N)$ and the IDS of the Sierpinski pre-gasket is purely discontinuous in accordance with Theorem 1 (ii).

[12] provides us with more explicit data:

$$
\begin{gathered}
\lambda_{1}=\frac{1}{N-1}, \quad \lambda_{2}=\frac{N+2}{2(N-1)}, \quad \lambda_{3}=\frac{N}{N-1}, \\
\hat{v}_{\lambda_{p}}^{(j)}=P_{\lambda_{p}}^{(j)}, \quad p=1,2, \quad j=1,2, \ldots,
\end{gathered}
$$

but, as for the allowed predecessors of $\lambda_{3}$,

$$
\hat{P}_{\lambda_{3}}^{(1)}=\left\{\frac{N}{2(N-1)}\right\} \subset\left\{\frac{1}{N-1}, \frac{N}{2(N-1)}\right\}=P_{\lambda_{3}}^{(1)}, \hat{P}_{\lambda_{3}}^{(j)}=P_{\frac{N}{2(N-1)}}^{(j-1)}, j=1,2, \ldots,
$$

so that

$$
M_{1, j}=M_{2, j}=2^{j}, M_{3, j}=2^{j-1} .
$$


Further

$$
r_{k}^{1}=\delta_{1, k}, r_{k}^{2}=\frac{N}{2}\left(N^{k-1}-2 N^{k-2}+1\right), \quad r_{k}^{3}=\frac{N}{2}\left(N^{k}-2 N^{k-1}+1\right) .
$$

Therefore

$$
\alpha_{1}=0, \quad \alpha_{2}=\frac{N-2}{N^{2}}, \quad \alpha_{3}=\frac{N-2}{N},
$$

and according to Theorem 1 (ii)

$$
\begin{aligned}
v & =\frac{N-2}{N^{2}} \sum_{j=0}^{\infty}\left(\frac{2}{N}\right)^{j} v_{\lambda_{2}}^{(j)}+\frac{N-2}{N}\left\{v_{\lambda_{3}}^{(0)}+\sum_{j=1}^{\infty} \frac{2^{j-1}}{N^{j}} v_{\frac{N}{2(N-1)}}^{(j-1)}\right\} \\
& =\frac{N-2}{N^{2}} \sum_{j=0}^{\infty}\left(\frac{2}{N}\right)^{j} v_{\lambda_{2}}^{(j)}+\frac{N-2}{N^{2}} \sum_{j=0}^{\infty}\left(\frac{2}{N}\right)^{j} v_{\frac{N}{2(N-1)}}^{(j)}+\frac{N-2}{N} \delta_{\lambda_{3}} .
\end{aligned}
$$

The above expression has appeared already in Fukushima-Shima [4, (6.1)].

Example 2 (modified Koch curve). In this case, $N=5: \Psi$ consists of 5 number of 3-similitudes. $N_{k}=\frac{3}{4}\left(5^{k}-1\right)$. By virtue of Molozemov [8], the spectral decimation works with

$$
\Psi(x)=\frac{2 x(3 x-4)(3 x-5)(x-1)}{2 x-3},
$$

so that $M=4(<5)$ and, according to Theorem 1 (ii), the IDS of the Laplacian on pre-fractal associated with this modified Koch curve is purely discontinuous. Moreover we know from [8] that

$$
\begin{gathered}
\lambda_{1}=\frac{5-\sqrt{13}}{6}, \quad \lambda_{2}=\frac{4}{3}, \quad \lambda_{3}=\frac{5+\sqrt{13}}{6}, \\
r_{k}^{p}=\frac{5^{k-1}+3}{4}, \quad a_{p}=\frac{1}{15}, \quad p=1,2,3,
\end{gathered}
$$

and

$$
\hat{P}_{\lambda_{p}}^{(j)}=P_{\lambda_{p}}^{(j)}, \quad M_{p, j}=4^{j}, \quad p=1,2,3, \quad j=1,2, \ldots .
$$

This case is simpler than the preceding example in that no prohibited predecessor of $\lambda_{p}$ exists. We get by Theorem 1 (ii) that

$$
v=\frac{1}{15} \sum_{p=1}^{3} \sum_{j=0}^{\infty}\left(\frac{4}{5}\right)^{j} v_{\lambda_{p}}^{(j)} .
$$

Example 3 (a real interval as a gasket). The interval $E=[0,1]$ can be viewed as a gasket for the 2-similitudes $\Phi=\left(\psi_{1}, \psi_{2}\right)$, where

$$
\psi_{1}(x)=\frac{1}{2} x, \psi_{2}(x)=\frac{1}{2}+\frac{1}{2} x
$$


Denote by $a, b, c, d, e$ the values of a function $u$ at the points $0,1, \frac{1}{2}, \frac{1}{4}, \frac{3}{4}$, respectively. The equation $\lambda u=-\mathrm{H}_{2} u$ now reads

$$
2 \lambda d=2 d-a-c, \quad 2 \lambda e=2 e-c-b, \quad 2 \lambda c=2 c-d-e .
$$

Solving the first two equations in $d$ and $e$ and substituting them into the third equation, we get

$$
4 \lambda(2-\lambda) c=2 c-a-b,
$$

from which we see that the spectral decimation works with

$$
\Phi(x)=2 x(2-x) .
$$

We easily see that

$$
\lambda_{1}=1, \quad r_{k}^{1}=1, \quad \mathscr{A}_{k+1}=\lambda_{1}+\Phi^{-1}\left(\mathscr{A}_{k}\right) \text { (disjoint sum). }
$$

Therefore the conditions of Theorem 1 (iii) are fulfilled with $N=M=2$ and $v$ equals the Brolin measure for $\Phi$. The Julia set of $\Phi$ is

$$
J=[0,2]
$$

because $\Phi$ is sent by the Möbius transform $x=-\frac{z}{2}+1$ into the polynomial $z^{2}-z$ which is known to possess the interval $[-2,2]$ as its Julia set (Theorem 12.1 of [1]). Accordingly

$$
\mathrm{d} v(x)=\frac{1}{\pi} \frac{I_{[0,2]}(x)}{\sqrt{x(2-x)}} d x .
$$

Indeed the logarithmic equilibrium measure of $J$ is characterized as a unique probability measure $\mu$ concentrated on $J$ such that

$$
U \mu(x)=C \text { q.e. } x \in J, U \mu(x) \leqq C \quad \forall x \in R^{2},
$$

for some constant $C$, where $U \mu(x)=\int_{R^{2}} \log \frac{1}{|x-y|} d \mu(y)$ the logarithmic potential of $\mu$. In this way, we recover the IDS

$$
\mathscr{N}(x)=\frac{1}{\pi}\left(\arcsin (x-1)+\frac{\pi}{2}\right), \quad 0 \leqq x \leqq 2
$$

for the discrete Laplacian $H u(k)=\frac{1}{2} u(k-1)+\frac{1}{2} u(k+1)-u(k)$ on $Z$.

\section{Tail Behaviours of IDS for the Nested Pre-fractal}

We denote by $H_{k}^{\prime}$ the generator of Lindstrøm random walk on $F^{(k)}\left(\sim \tilde{F}^{(k)}\right)$ reflected at the boundary $F\left(\sim \alpha^{k} F\right)$. That is

$$
H_{k}^{\prime} u(\xi)=\sum_{\eta \in F^{(k)}} p_{\xi \eta}^{(k)}(u(\eta)-u(\xi)), \quad \xi \in F^{(k)} .
$$

Define $v_{k}^{\prime}$ in the same way as $v_{k}$. For $v_{k}$ and $v_{k}^{\prime}$, let $\mathscr{N}_{k}(x)=v_{k}([0, x])$, and $\mathscr{N}_{k}^{\prime}(x)=v_{k}^{\prime}([0, x])$. 
Using the min-max principle in the same way as [3], we can easily get the following inequalities: Let $N_{k}^{\prime}$ be the cardinality of the set $F^{(k)}$,

$$
\begin{gathered}
0 \leqq N_{k}^{\prime} \mathscr{N}_{k}^{\prime}(x)-N_{k} \mathscr{N}_{k}(x) \leqq N_{0}^{\prime} \\
N N_{k-1} \mathscr{N}_{k-1}(x) \leqq N_{k} \mathscr{N}_{k}(x), \\
N N_{k-1}^{\prime} \mathscr{N}_{k-1}^{\prime}(x) \geqq N_{k}^{\prime} \mathscr{N}_{k}^{\prime}(x) .
\end{gathered}
$$

By iterating (14) and (15), we are then led to,

$$
\frac{N_{l}}{N^{l}} \mathscr{N}_{l}(x) \leqq \frac{N_{k}}{N^{k}} \mathscr{N}_{k}(x) \leqq \frac{N_{k}^{\prime}}{N^{k}} \mathscr{N}_{k}^{\prime}(x) \leqq \frac{N_{l}^{\prime}}{N^{l}} \mathscr{N}_{l}^{\prime}(x), k>l .
$$

Thus $\left\{\frac{N_{k}}{N^{k}} \mathscr{N}_{k}(x)\right\}\left(\right.$ resp. $\left.\left\{\frac{N_{k}^{\prime}}{N^{k}} \mathscr{N}_{k}^{\prime}(x)\right\}\right)$ increases (resp. decreases) as $k \uparrow$. Noting that $N_{k}=\left(N^{k}-1\right) N_{1} /(N-1), N_{k}^{\prime}=N_{k}+N_{0}^{\prime}$,

$$
\frac{N-1}{N_{1}} \frac{N_{l}}{N^{l}} \mathscr{N}_{l}(k) \leqq \lim _{k \rightarrow \infty} \mathscr{N}_{k}(x) \leqq \lim _{k \rightarrow \infty} \mathscr{N}_{k}^{\prime}(x) \leqq \frac{N-1}{N_{1}} \frac{N_{l}^{\prime}}{N^{l}} \mathscr{N}_{l}^{\prime}(x)
$$

Furthermore by (13),

$$
0 \leqq \frac{N_{l}^{\prime}}{N^{l}} \mathcal{N}_{l}^{\prime}(x)-\frac{N_{l}}{N^{l}} \mathcal{N}_{l}(x) \leqq \frac{N_{0}^{\prime}}{N^{l}},
$$

and the above limits must coincide. The right continuous modification of the limiting function is the IDS $\mathscr{N}(x)$. Indeed, at any continuous point,

$$
1-\frac{N-1}{N_{1}} \frac{N_{l}^{\prime}}{N^{l}} \mathscr{N}_{l}^{\prime}(x) \leqq 1-\mathscr{N}(x) \leqq 1-\frac{N-1}{N_{1}} \frac{N_{l}}{N^{l}} \mathscr{N}_{l}(x),
$$

from which follows $\lim _{x \rightarrow \infty} \mathscr{N}(x)=1$. We summarize those facts as follows:

Lemma 1. $v_{k}$ and $v_{k}^{\prime}$ converge as $k \rightarrow \infty$ to a same probability measure $v$. Furthermore, we have for $\mathscr{N}(x)=v([0, x])$,

$$
\frac{N-1}{N_{1}} \frac{N_{l}}{N^{l}} \mathscr{N}_{l}(x) \leqq \mathscr{N}(x) \leqq \frac{N-1}{N_{1}} \frac{N_{l}^{\prime}}{N^{l}} \mathscr{N}_{l}^{\prime}(x)
$$

for all $l$.

Let $c$ be the probability for the Lindstrøm random walk on $F^{(1)}$ of starting at a boundary point and returning to it before reaching other boundary points, and let $\mu_{k}$ be the discrete measure on $F^{(k)}$ such as

$$
\mu_{k}(x)=\#\left\{\left(i_{1}, \ldots, i_{k}\right) ; F_{i_{1}} \cdots i_{k} \ni x, 1 \leqq i_{1}, \ldots, i_{k} \leqq N\right\} .
$$

Clearly $\mu_{k}\left(F^{(k)}\right)=N_{0}^{\prime} N^{k}$. We then define the constant $C$ as follows:

$$
\begin{aligned}
C= & \inf \left\{(1-c)^{-k}\left(-H_{k}^{\prime} f, f\right)_{k} ; f \text { is any real valued function on } F^{(k)}\right. \\
& \text { such as } \left.\max \left\{|f(x)-f(y)|^{2} ; x, y \in F^{(k)}\right\}=1, k=1,2, \ldots\right\},
\end{aligned}
$$

where $(\cdot, \cdot)_{k}$ stands for the inner product weighted by $\mu_{k}$. It was shown in [6] that $C$ is finite and strictly positive. 
Lemma 2. Let $\lambda_{k}$ be the first eigenvalue of $-H_{k}$ and $\lambda_{k}^{\prime}$ the second eigenvalue of $-H_{k}^{\prime}$. Then,

$$
\lambda_{k}^{\prime} \geqq \frac{C}{N_{0}^{\prime}}\left(\frac{1-c}{N}\right)^{k},
$$

and there exist $k_{0}$ and a constant $C^{\prime}>0$ such that

$$
\lambda_{k} \leqq\left(\frac{1-c}{N}\right)^{k} C^{\prime} \text { for all } k \geqq k_{0} .
$$

Proof. Let $L_{k}$ be the orthogonal complement to the eigenspace of the first eigenvalue of $-H_{k}^{\prime}$. It is then easy to see that $L_{k}$ is the totality of the real valued function on $F^{(k)}$ such that $\int_{F^{(k)}} f d \mu_{k}=0$. For any $f \in L_{k}$, it hence holds that

$$
\begin{aligned}
(f, f)_{k} & =\int_{F^{(k)}}\left\{\frac{1}{N_{0}^{\prime} N^{k}} \int_{F^{(k)}}(f(x)-f(y)) \mu_{k}(d y)\right\}^{2} \mu_{k}(d x) \\
& \leqq N_{0}^{\prime} N^{k} \max _{x, y \in F^{(k)}}\left\{|f(x)-f(y)|^{2}\right\} .
\end{aligned}
$$

In view of the definition of $C$,

$$
C \leqq \frac{N_{0}^{\prime} N^{k}}{(1-c)^{k}} \inf \left\{\frac{\left(-H_{k}^{\prime} f, f\right)_{k}}{(f, f)_{k}} ; f \in L_{k}\right\},
$$

from which (17) follows.

Consider the nested fractal $E$ corresponding to the nested pre-fractal $\tilde{F}^{\infty}$. Let $\mu$ be the $\log N / \log \alpha$-dimensional normalized Hausdorff measured on $E$. We denote by $(\mathscr{E}, \mathscr{D}(\mathscr{E}))$ the Dirichlet form on $L^{2}(E ; d \mu)$ defined in [6], where it was shown that $\mathscr{D}(\mathscr{E})$ is a subspace of the space of continuous functions on $E$, and that $\left\{(1-c)^{-k}\left(-\left.H_{k}^{\prime} f\right|_{F^{(k)}},\left.f\right|_{F^{(k)}}\right)_{k}\right\}$ increases as $k \uparrow \infty$ to $\mathscr{E}(f, f)$ for all $f \in \mathscr{D}(\mathscr{E})$. We thus get that, for a fixed $f \in \mathscr{D}(\mathscr{E})$ vanishing at the boundary and not identically zero,

$$
\begin{aligned}
& \lambda_{k} \leqq \frac{\left(-\left.H_{k} f\right|_{\left.F^{(k)},\left.f\right|_{F^{(k)}}\right)_{k}}\right.}{\left(\left.f\right|_{\left.F^{(k)},\left.f\right|_{F^{(k)}}\right)_{k}}\right.}=\left(\frac{1-c}{N}\right)^{k} \frac{(1-c)^{-k}\left(-\left.H_{k} f\right|_{F^{(k)},\left.f\right|_{\left.F^{(k)}\right)_{k}}}\right.}{N^{-k}\left(\left.f\right|_{\left.F^{(k)},\left.f\right|_{F^{(k)}}\right)_{k}}\right.} \\
& \leqq\left(\frac{1-c}{N}\right)^{k} \frac{\mathscr{E}(f, f)}{N^{-k}\left(\left.f\right|_{\left.F^{(k)},\left.f\right|_{F^{(k)}}\right)_{k}}\right.} .
\end{aligned}
$$

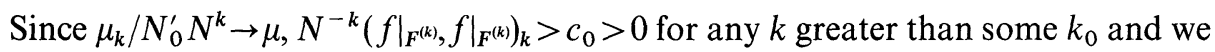
arrive at (18).

\section{Theorem 2.}

$$
0<\lim _{x \downarrow 0} \inf _{0} \frac{\mathscr{N}(x)}{x^{d_{s} / 2}} \leqq \lim _{x \downarrow 0} \sup \frac{\mathscr{N}(x)}{x^{d_{s} / 2}}<\infty .
$$

Proof. For any $x \in\left(0,\left(\frac{1-c}{N}\right)^{k_{0}} C^{\prime}\right)$, let $k>k_{0}$ be the positive integer such as

$$
\left(\frac{1-c}{N}\right)^{k} C^{\prime} \leqq x<\left(\frac{1-c}{N}\right)^{k-1} C^{\prime}
$$


We then have $\mathscr{N}_{k}(x) \geqq 1 / N_{k}$ by (18). By virtue of the inequality (16), we get that

$$
\mathscr{N}(x) \geqq \frac{N-1}{N_{1}} \frac{N_{k}}{N^{k}} \mathscr{N}_{k}(x) \geqq \frac{N-1}{N_{1}} \frac{1}{N^{k}} .
$$

Since the spectral dimension $d_{s}$ of the nested fractal $E$ is equal to $2 \log N /$ $(\log N-\log (1-c))($ see $[2,7])$, we are led to

$$
\mathscr{N}(x) \geqq \frac{N-1}{N_{1} N} C^{\prime-d_{s} / 2} x^{d_{s} / 2} .
$$

Similarly we get the following upper estimate from (17): that is, for $x \in$ $\left(0, \frac{C}{N_{0}^{\prime}}\left(\frac{1-c}{N}\right)\right)$

$$
\mathscr{N}(x) \leqq \frac{(N-1) N}{N_{1}}\left(\frac{C}{N_{0}^{\prime}}\right)^{-d_{s} / 2} x^{d_{s} / 2}
$$

\section{References}

1. Brolin, H.: Invariant sets under iteration of rational functions. Ark. Mat. 6, 103-144 (1965)

2. Fukushima, M.: Dirichlet forms, diffusion processes and spectral dimensions for nested fractals. In: Albeverio, S., Fenstad, J.E., Holden, H., Lindstrøm, T. (eds.), Ideas and Methods in Mathematical Analysis, Stochastics, and Applications, Vol. 1, Cambridge: Cambridge Univ. Press, 1992, pp. 151-161

3. Fukushima, M., Nakao, S., Kotani, S.: Random spectrum. Seminar on Probability, Vol. 45, 1977 (in Japanese)

4. Fukushima, M., Shima, T.: On a spectral analysis for the Sierpinski gasket. Potential Analysis 1, 1-35 (1992)

5. Kigami, J., Lapidus, M.L.: Weyl's problem for the spectral distributions of Laplacians on p.c.f self-similar fractals. Commun. Math. Phys. 158, 93-125 (1993)

6. Kusuoka, S.: Diffusion processes on nested fractals. Lecture Notes in Math. vol. 1567, Springer 1993

7. Lindstrøm, T.: Brownian motion on nested fractals. Memoir AMS420, 1989

8. Malozemov, L.: The difference Laplacian $\Delta$ on the modified Koch curve. Russ. J. Math. Phys. 3, no. 1 (1992)

9. Malozemov, L.: The integrated density of states for the difference Laplacian on the modified Koch graph. Commun. Math. Phys. 156, 387-397 (1993)

10. Rammal, R.: Spectrum of harmonic excitations on fractals. J. Physique 45, 191-204 (1984)

11. Rammal, R., Toulouse, G.: Random walks on fractal structures and percolation clusters. J. Physique Lett. 43, L13-L22 (1982)

12. Shima, T.: On eigenvalue problems for the random walks on the Sierpinski pre-gaskets. Japan J. Indus. Appl. Math. 8, 127-141 (1991)

13. Shima, T.: Lifschitz tails for random Schrödinger operators on nested fractals. Osaka J. Math. 29, 749-770 (1992) 
